\title{
A SHORT PROOF OF RIGIDITY OF CONVEX POLYTOPES
}

\author{
IGOR PAK
}

\begin{abstract}
We present a much simplified proof of Dehn's theorem on the infinitesimal rigidity of convex polytopes. Our approach is based on the ideas of Trushkina 11] and Schramm [10.
\end{abstract}

\section{Introduction}

Let $P \subset \mathbb{R}^{3}$ be a simplicial convex polytope. Define a continuous deformation $\left\{P_{t}\right.$ : $t \in[0,1]\}$ of $P=P_{0}$ to be a family of convex polytopes with the same combinatorial structure, the same corresponding edge lengths, and continuity on vertices. We say that $P$ is continuously rigid if every such deformation is a rigid motion in $\mathbb{R}^{3}$. It is a classical corollary from the Cauchy theorem that every simplicial polytope $P$ is continuously rigid (see below). In this article we present a simple proof of Dehn's theorem, which also implies the continuous rigidity.

Let $\boldsymbol{v}_{i}(t)=\overrightarrow{O v_{i}}$ be a vector from the origin $O$ into the vertex $v_{i}$ in $P_{t}$. Think of vectors $\boldsymbol{v}_{i}^{\prime}(t)$ as of velocities of vertices $v_{i}$. For an edge length $\left|v_{i} v_{j}\right|$ to be constant under the deformation we need $\left\|\boldsymbol{v}_{i}(t)-\boldsymbol{v}_{j}(t)\right\|^{\prime}=0$, where $\|\boldsymbol{w}\|=(\boldsymbol{w}, \boldsymbol{w})=|\boldsymbol{w}|^{2}$. Thus, in particular, at $t=0$ we have:

$$
\begin{aligned}
0 & =\frac{d}{d t}\left\|\boldsymbol{v}_{i}(t)-\boldsymbol{v}_{j}(t)\right\|_{t=0}=\frac{d}{d t}\left\|\left(\boldsymbol{v}_{i}(0)-\boldsymbol{v}_{j}(0)\right)+t\left(\boldsymbol{v}_{i}^{\prime}(0)-\boldsymbol{v}_{j}^{\prime}(0)\right)\right\|_{t=0} \\
& =2\left(\boldsymbol{v}_{i}(0)-\boldsymbol{v}_{j}(0), \boldsymbol{v}_{i}^{\prime}(0)-\boldsymbol{v}_{j}^{\prime}(0)\right) .
\end{aligned}
$$

This leads to the following definition of infinitesimal rigidity.

Let $V=\left\{v_{1}, \ldots, v_{n}\right\}$ and $E$ be the set of vertices and edges of $P$. We will assume that vertices $v_{1}, v_{2}$ and $v_{3}$ form a triangular face of $P$; they are called base vertices, and face $\left(v_{1} v_{2} v_{3}\right)$ is called base triangle. Suppose we are given a vector $\boldsymbol{a}_{i} \in \mathbb{R}^{3}$, for every $i \in\{1, \ldots, n\}$. We say that the set of vectors $\left\{\boldsymbol{a}_{i}, 1 \leq i \leq n\right\}$ defines an infinitesimal rigid motion if

$$
(*) \quad\left(\boldsymbol{v}_{i}-\boldsymbol{v}_{j}, \boldsymbol{a}_{i}-\boldsymbol{a}_{j}\right)=0, \text { for every }\left(v_{i}, v_{j}\right) \in E .
$$

An infinitesimal rigid motion is called planted if the base velocities vectors are equal to zero: $\boldsymbol{a}_{1}=\boldsymbol{a}_{2}=\boldsymbol{a}_{3}=\mathbf{0}$. Finally, we say that a simplicial polytope $P \subset \mathbb{R}^{3}$ is infinitesimally rigid if every planted infinitesimal rigid motion is trivial: $\boldsymbol{a}_{i}=\mathbf{0}$, for all $i \in\{1, \ldots, n\}$. Here is the main result of this paper:

Dehn's Theorem. Every simplicial convex polytope in $\mathbb{R}^{3}$ is infinitesimally rigid.

Date: 11 August 2005.

Key words and phrases. Convex polytope, infinitesimal rigidity, Dehn's theorem. 
Of course, the restriction to planted infinitesimal rigid motions is necessary, as the usual rigid motions of $P$ in $\mathbb{R}^{3}$ can define nontrivial infinitesimal rigid motions. By the argument above, the infinitesimal rigidity implies the continuous rigidity:

Corollary (Cauchy). Every simplicial convex polytope in $\mathbb{R}^{3}$ is continuously rigid.

In this paper we present a new proof of Dehn's theorem, based on the approach by Trushkina [11] (see also a followup [12]). Unfortunately, the technical details in Trushkina's paper are somewhat complicated. We substitute Trushkina's definition of the inversion with the one given by Schramm [10] (for different purposes). We should mention an important survey [4] (see §4.6), which helped us to translate the ideas in [10] into the language of infinitesimal rigidity.

Before we conclude, let us repeat that the continuous rigidity of convex polytopes follows from the classical Cauchy theorem [1, 3, 4]. Dehn's theorem was established by Dehn in [5] in an equivalent language of the static rigidity. This result became fundamental in the modern study of rigidity of frameworks and non-convex polyhedra [4, 14, and a number of proofs have been found. We refer to [2, 8] for the exposition of Dehn's original proof, to [6, 7, 9, 13] for applications and modern treatment, and to [2, 4] for further references.

\section{Proof of Infinitesimal Rigidity}

First, note that equations $(*)$ above say that the difference in velocities along an edge is orthogonal to this edge of the polytope. Think of velocity vectors as vector functions on vertices of $P$ which are equal to $\mathbf{0}$ on base vertices $v_{1}, v_{2}, v_{3}$. The idea of the proof is to enlarge the set of such functions and prove a stronger result.

As before, let $V=\left\{v_{1}, \ldots, v_{n}\right\}$ and $E$ be the set of vertices and edges of a simplicial convex polytope $P \subset \mathbb{R}^{3}$. Consider the set of all vector sequences $\left(\boldsymbol{a}_{1}, \ldots, \boldsymbol{a}_{n}\right)$, $\boldsymbol{a}_{i} \in \mathbb{R}^{3}$, such that for every edge $\left(v_{i}, v_{j}\right) \in E$ we have one of the following three possibilities:

1. $\left(\boldsymbol{v}_{i}-\boldsymbol{v}_{j}, \boldsymbol{a}_{i}\right)=\left(\boldsymbol{v}_{i}-\boldsymbol{v}_{j}, \boldsymbol{a}_{j}\right)=0$,

2. $\left(\boldsymbol{v}_{i}-\boldsymbol{v}_{j}, \boldsymbol{a}_{i}\right)<0$ and $\left(\boldsymbol{v}_{i}-\boldsymbol{v}_{j}, \boldsymbol{a}_{j}\right)<0$,

3. $\left(\boldsymbol{v}_{i}-\boldsymbol{v}_{j}, \boldsymbol{a}_{i}\right)>0$ and $\left(\boldsymbol{v}_{i}-\boldsymbol{v}_{j}, \boldsymbol{a}_{j}\right)>0$.

In other words, we require that projections of velocity vectors $\boldsymbol{a}_{i}$ and $\boldsymbol{a}_{j}$ onto edge $\left(v_{i}, v_{j}\right)$ have the same signs. We say that a vertex $v_{i}$ is dead if $\boldsymbol{a}_{i}=\mathbf{0}$; it is live otherwise. We need to prove that for every vector sequence $\left(\boldsymbol{a}_{1}, \ldots, \boldsymbol{a}_{n}\right)$ as above, if the base vertices are dead, then all vertices $v_{i} \in V$ are dead. By definition of the infinitesimal rigidity, this would immediately imply the theorem.

Denote by $\Gamma=(V, E)$ the graph of $P$. Since $P$ is simplicial, $\Gamma$ is a plane triangulation. Consider an orientation of edges of $\Gamma$ in the direction of projections of the velocity vectors. More precisely, we orient edges $v_{i} \rightarrow v_{j}$ in case $\mathbf{2}$, orient them $v_{i} \leftarrow v_{j}$ in case $\mathbf{3}$, and leave them unoriented in case $\mathbf{1}$. Clearly, the edges adjacent to dead vertices are unoriented.

Consider two edges $e=\left(v_{i}, v_{j}\right)$ and $e^{\prime}=\left(v_{i}, v_{r}\right), e, e^{\prime} \in E$, with a common vertex $v_{i}$, such that $\left(v_{i} v_{j} v_{r}\right)$ is a face in $P$. We say that edges $e$ and $e^{\prime}$ 
- have one inversion if one of them is oriented into $v_{i}$, and the other out of $v_{i}$,

- have zero inversions if both of them are oriented into $v_{i}$ or out of $v_{i}$,

- have a half-inversion if one of the edges is oriented and the other is unoriented,

- have one inversion if both of them are unoriented and $v_{i}$ is a live vertex,

- have zero inversions if both of them are unoriented and $v_{i}$ is a dead vertex.

When we talk about the number of inversions in a subgraph, in a triangle, or around a vertex, we mean the total sum of inversions between pairs of edges involved. For example, we say that a graph has at least $q$ inversions if this sum is $\geq q$.

A triangle is called active if at least one of its vertices is live; it is called inactive otherwise. Now consider different orientations of an active triangle $\left(v_{i} v_{j} v_{r}\right)$ where vertex $v_{i}$ is live (see some of them in Figure 11). A simple enumeration of all possible cases gives the following result:

Lemma 1. Every active triangle has at least one inversion.
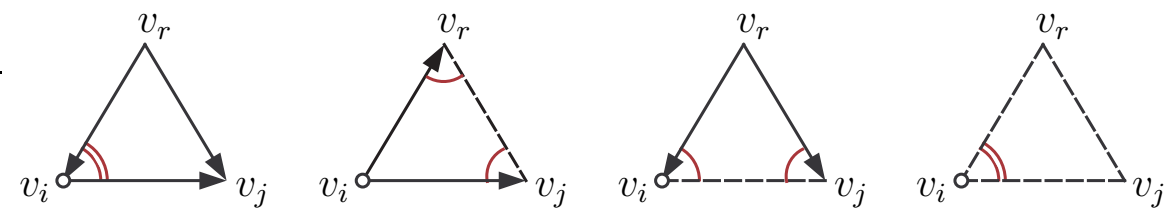

FiguRE 1. Different orientations of $\left(v_{i} v_{j} v_{r}\right)$, where vertex $v_{i}$ is live.

This lemma gives a lower bound on the number of inversions in $\Gamma$. To get an upper bound, we count inversions around vertices:

Lemma 2. There are at most two inversions around every live vertex.

We postpone the proof of the lemma until we finish the proof of the theorem. Consider what this gives us when the only unoriented edges in $\Gamma$ are the base edges $\left(v_{1}, v_{2}\right),\left(v_{1}, v_{3}\right)$, and $\left(v_{2}, v_{3}\right)$. In this case we have $(n-3)$ live vertices. Recall also that there are no inversions around dead vertices. Thus, by Lemma 2, there are at most $2(n-3)=2 n-6$ inversions in $\Gamma$. On the other hand, recall that a triangulation with $n$ vertices always has $(2 n-4)$ triangles (see e.g. 3]), and, by assumption, only one of them is inactive. Thus, by Lemma 1 , there are at least $(2 n-5)$ inversions in $\Gamma$, a contradiction.

We use the same strategy in general case. Remove from $\Gamma$ all inactive triangles together with all edges and vertices which belong only to inactive triangles. Denote by $H=\left(V^{\prime}, E^{\prime}\right)$ a connected component of the remaining graph. Since $\Gamma$ is planar, the induced subgraph $H$ of $\Gamma$ has a well defined boundary $\partial H$. Denote by $k$ the number of vertices in $\partial H$ (all of them dead), and by $\ell$ the number of connected components of $\partial H$. Finally, denote by $m$ the number of vertices in $H \backslash \partial H$ (some of them live and some possibly dead). By Lemma 2 , there are at most $2 m$ inversions in $H$.

Let us now estimate the number of inversions via the number $t$ of triangles in $H$. Observe that the total number of vertices and edges in $H$ is given by

$$
\left|V^{\prime}\right|=m+k, \quad \text { and } \quad 2\left|E^{\prime}\right|=k+3 t .
$$


On the other hand, there are $f=(t+\ell)$ faces in $H$ taken together with the boundary components. Substituting these values into Euler's formula $\left|V^{\prime}\right|-\left|E^{\prime}\right|+f=2$, we conclude that graph $H$ has exactly $t=2 m+k+2 \ell-4$ triangles. Since there is at least one inactive triangle $\left(v_{1} v_{2} v_{3}\right)$, we have $k \geq 3$ and $\ell \geq 1$. Therefore, by Lemma 1 , there are at least

$$
t=2 m+k+2 \ell-4 \geq 2 m+3+2-4=2 m+1
$$

inversions in $H$, a contradiction.

\section{Proof of Lemma 2.}

Let us consider all possibilities one by one, and check the claim in each case. Suppose a vertex $v_{i}$ is adjacent to three or more unoriented edges. This means that $\boldsymbol{a}_{i}$ is orthogonal to at least three vectors spanning $\mathbb{R}^{3}$. Therefore, $\boldsymbol{a}_{i}=\mathbf{0}$ and $v_{i}$ is a dead vertex with zero inversions.

Suppose now that $v_{i}$ is adjacent to exactly two unoriented edges $e, e^{\prime}$ in $P$. This means that $\boldsymbol{a}_{i} \neq \mathbf{0}$ is orthogonal to a plane spanned by these edges. Observe that $e, e^{\prime}$ separate the edges oriented into $v_{i}$ from those oriented out of $v_{i}$. Thus, there are either two half-inversions and one inversion if the edges $e, e^{\prime}$ are adjacent, or four half-inversions if $e, e^{\prime}$ are not adjacent (see Figure 2).
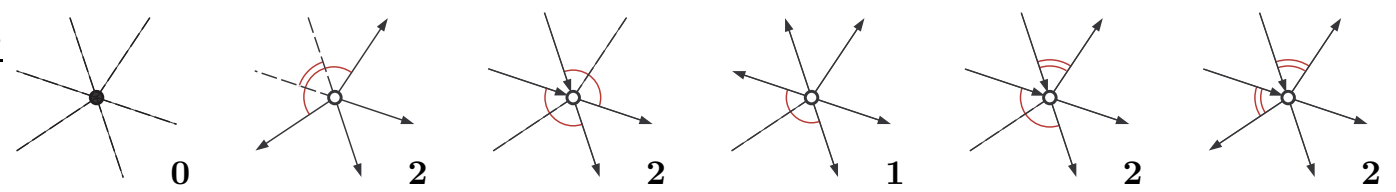

FiguRE 2. The number of inversions around a vertex in different cases.

Next, suppose that $v_{i}$ is adjacent to exactly one unoriented edge $e$. Since $\boldsymbol{a}_{i} \neq \mathbf{0}$ in this case, consider a plane containing $v_{i}$ and orthogonal to $\boldsymbol{a}_{i}$. This plane contains $e$ and separates the edges oriented into $v_{i}$ from those oriented out of $v_{i}$. Therefore, there are either two half-inversions if all other edges are oriented into $v_{i}$, or out of $v_{i}$, or two half-inversions and one inversion otherwise.

Finally, if $v_{i}$ is a live vertex that is not adjacent to any unoriented edges, then the plane orthogonal to $\boldsymbol{a}_{i}$ separates the edges into two parts: those oriented into $v_{i}$ from those oriented out of $v_{i}$. Thus, there are exactly two inversions in this case.

Final remarks. Let us note that Dehn's theorem and Cauchy's corollary hold in the generality of all convex polytopes. The reduction to the simplicial case is straightforward: triangulate the surface of $P$ by adding diagonals of the faces. We skip the details. We should mention also that Dehn's original proof is also based on a graph-theoretic argument, very much different from the one presented here.

The proof we presented above easily splits into two parts: global and local, not unlike the original proof of the Cauchy theorem (see e.g. 3, 4]). The local part (Lemma 2), while different, has the same flavor as the the sign changes lemma in 
Cauchy's proof. It is even more similar to the local part of other proofs of Dehn's theorem (see [4, 8, 13]). The global part is a graph-theoretic argument somewhat similar in style and complexity to the double counting argument used in the proof of the Cauchy theorem, with both arguments based on Euler's formula.

Finally, let us mention that the result of Trushkina is a bit stronger as she allows the base vertices not to be on the same face. When applied to the infinitesimal rigidity of polytopes, this gives an equivalent result, but the graph-theoretic claim becomes harder to prove. Similarly, Schramm proves a much stronger result about certain delicate graph labeling [10]. Interestingly, at some point he employs a counting angles argument, essentially reproving Euler's formula.

Acknowledgements. I am grateful to Bob Connelly and Oded Schramm for their comments on article [10, and to the NSF for the financial support. I am also indebted to Victor Alexandrov for his interest in the paper and remarks on the presentation. Last but not least, I would like to thank Susanna Yusufova for her love and support.

\section{REFERENCES}

[1] A. D. Aleksandrov, Vypuklye mnogogranniki (in Russian), M.: Gostekhizdat, 1950; English translation: Convex polyhedra, Springer, Berlin, 2005.

[2] V. A. Alexandrov, Inverse function theorems and their applications to the theory of polyhedra (in Russian), to appear in Reviews in Mathematics and Mathematical Physics (2005), 125 pp.; available electronically at http://math.nsc.ru/ vaalex

[3] M. Berger, Géométrie, Vol. 1-5, (in French), Nathan, CEDIC, Paris, 1977.

[4] R. Connelly, Rigidity, in Handbook of Convex Geometry, vol. A, 223-271, North-Holland, Amsterdam, 1993.

[5] M. Dehn, Über die Starreit konvexer Polyeder, Math. Ann. 77 (1916), 466-473; available electronically at http://dz-srv1.sub.uni-goettingen.de/cache/toc/D37460.html

[6] M. Fedorchuk and I. Pak, Rigidity and polynomial invariants of convex polytopes, Duke Math. J. 129 (2005), 371-404; available electronically at http://www-math.mit.edu/ ${ }^{\sim}$ pak

[7] H. Gluck, Almost all simply connected closed surfaces are rigid, in Lecture Notes in Math. 438, 225-239, Springer, Berlin, 1975.

[8] I. Pak, Lectures on Combinatorial Geometry and Convex Polytopes, monograph in preparation.

[9] B. Roth, Rigid and flexible frameworks, Amer. Math. Monthly 88 (1981), no. 1, 6-21.

[10] O. Schramm, How to cage an egg, Invent. Math. 107 (1992), 543-560; available electronically at http://dz-srv1.sub.uni-goettingen.de/cache/toc/D183703.html

[11] V. I. Trushkina, A coloring theorem and the rigidity of a convex polyhedron (in Russian), Ukrainian Geometric Sbornik No. 24 (1981), 116-122.

[12] V. I. Trushkina, Method of 3-colouring of graphs, Sib. Math. J. 28 (1987), 331-342.

[13] W. Whiteley, Infinitesimally rigid polyhedra. I. Statics of frameworks, Trans. AMS 285 (1984), 431-465.

[14] W. Whiteley, Rigidity and scene analysis, in Handbook of discrete and computational geometry, 893-916, CRC Press, Boca Raton, FL, 1997.

\section{Igor Pak}

M.I.T. Department of Mathematics

Cambridge, MA 02139

U.S.A.

pak@math.mit.edu 